\title{
Erratum
}

Qing Sun, Yanli Chen, Fuxue Li, Minghong Jia*, Guoqing Shi*

\section{Erratum to: A one-step incubation ELISA kit for rapid determination of dibutyl phthalate in water, beverage and liquor}

https://doi.org/10.1515/chem-2020-0555

received August 26, 2020; accepted August 26, 2020

Erratum to: Quing Sun, Yanli Chen, Fuxue Li, Minghong Jia and Guoqing Shi. July 2019. A one-step incubation ELISA kit for rapid determination of dibutyl phthalate in water, beverage and liquor. Open Chemistry. Volume 17, pages 392-400. (DOI: 10.1515/ chem-2019-0052):

The first name of the first author Qing Sun was misspelled as Quing. For the readers' convenience the corrected authors' list appears as follows:

Qing Sun, Yanli Chen, Fuxue Li, Minghong Jia and Guoqing Shi

* Corresponding author: Minghong Jia, Beijing Key Laboratory of Detection and Control of Spoilage Organisms and Pesticide Residues in Agricultural Products-Beijing University of Agriculture, Beijing, 102206, China, e-mail: mh_jia@yahoo.com

* Corresponding author: Guoqing Shi, School of Chemistry and Biological Engineering, University of Science and Technology Beijing, Beijing, 100083, China; Beijing Key Laboratory of Detection and Control of Spoilage Organisms and Pesticide Residues in Agricultural Products-Beijing University of Agriculture, Beijing, 102206, China, e-mail: shiguoqing@ustb.edu.cn

Qing Sun, Yanli Chen, Fuxue Li: School of Chemistry and Biological Engineering, University of Science and Technology Beijing, Beijing, 100083, China 